\title{
Modeling and Real-time Control of Laser Metal Additive Manufacturing
}

\author{
Amir Khajepour \\ Professor and Canada Research Chair \\ University of Waterloo \\ Department of Mechanical and Mechatronics Engineering \\ Waterloo, Ontario, CANADA
}

Laser metal additive manufacturing is an advanced laser materials processing technique that is used in coating, repair, prototyping, and manufacturing of complex parts. A laser beam melts powder particles and a thin layer of the substrate to create an added layer that is directly bonded to the substrate. In this process, variety of materials can be deposited on a substrate to form a layer with a thickness of under $2 \mathrm{~mm}$. Thicker depositions and 3-D parts are produced by a successive layering technique.

The laser metal additive technology is a complex process in which many parameters have significant impact on the deposited materials. The laser power, beam quality, laser focal point, deposition speed, powder size, feed rate, substrate material, and substrate temperature are examples of effective parameters. These parameters can affect considerably the microstructure, porosity, hardness, and crack in the deposited material. In this talk, we discuss the process and the importance of modeling and real time control in bringing the technology to mainstream manufacturing. 